\title{
PIV MEASUREMENTS OF FLOW STRUCTURES CREATED BY A PULSATING FLAME SPREAD OVER 1-PROPANOL
}

\author{
M. I. Hassan ${ }^{1}$, K. Kuwana ${ }^{1}$, K. Saito ${ }^{1}$, and T. Hirano ${ }^{2}$ \\ 1 Department of Mechanical Engineering, \\ University of Kentucky, \\ Lexington, KY, USA \\ 2 National Research Institute of Fire and Disaster, \\ Tokyo, Japan
}

\begin{abstract}
Particle image velocimetry (PIV) was applied to measure detailed flow structures both at the gas and liquid phases created by a pulsating flame spread over 1-propanol, placed in a narrow wide long tray. The PIV measurements revealed a small flow circulations in both gas and liquid phases just ahead of the spreading flame. Similar measurements were done earlier by Laser Sheet Particle Tracking (LSPT) technique, but LSPT data were rather qualitative and limited numbers of particles were tracked due to its manual count system. The current PIV can compensate these shortcomings with its automated particle tracking and data processing system. With PIV system that was focused on two critical steps, just before and after flame jumping, we obtained more detailed 2-D flow fields than the LSPT and successfully obtained quantified flow vector fields. As a result, this study confirmed the earlier LSPT result that the existence of two opposite vortices in both liquid and gas phases just before the flame jumping. In the stage just after the flame jumping, however, PIV found a small circulation in the liquid phase and a weak circulation in the gas phase, both of which were not reported in our earlier study.
\end{abstract}

Key words: flame spread, PIV, pool fire

\section{INTRODUCTION}

When a flame spreads over the surface of a liquid fuel whose initial temperature is kept below its flash point, a flame-induced flow may occur in the gas and liquid phases. The study of the flame-induced flow has been an important subject for the fire safety researchers who have been involved in fire safety and control aspects of spreading fires over liquid fuels. To develop an effective fire control and prevention system for liquid storage tanks and containers, the structure of the flame-induced flow (both at liquid and gas phases) and its role to flame spreading process needs to be understood and they have been a central issue on fire research [1-5].

When the initial temperature of liquid is above its flash point, flame spreads through the gas phase where flammable fuel-air mixture already exists. When the initial temperature is below the flash point, then the liquid phase starts to play an important role in spreading. Flame spreads uniformly when the initial liquid temperature is slightly below the flash point. With a further decrease of the liquid temperature, the flame starts to 
spread with pulsating motion, called pulsating spread. With a further decrease of the liquid temperature beyond the pulsating spread temperature, the rate of flame spread becomes so low and the spread occurs with a very low frequency pulsation, called as pseudo-uniform spread [6].

The existence of recirculation cell in both gas and liquid phases was predicted theoretically [7-9] and observed experimentally [10, 11]. These recirculation cells were found to play an important role in flame spread. However, quantitative measurements of liquid and gas velocity have met little success due to the difficulty associated with the nature of flame-internal flow structure which is higly transient and in the order of submillimeter.

Previously we applied five different techniques (laser sheet particle tracking, infrared thermograph, dual wave-length holographic interferometer, smoke tracing, and high speed photography) to simultaneously measure three-dimensional structures of flameinduced flow both at liquid and gas phases, the liquid surface temperature and the fuel vapor concentration over the fuel surface, all that were created during a pulsating flame spread over n-propanol. In that measurement, we applied our own laser sheet particle tracking (LSPT) system and manually tracked approximately 20 individual particles. LSPT can work better for limited number particles, less than 50, but may start to lose its accuracy when a larger number of particles are involved mainly because of its manual count procedure.

To overcome this shortcoming, recently we obtained an integrated particle image velocimetry (PIV), conducted a series of PIV measurements and compared its results to the LSPT measurements. In comparison to the manually operated LSPT, however, our PIV system has an automated integrated data recording/processing system that allows us to track larger number of particles with much faster processing rate. With PIV, we expect to see more detailed flow structures which can be used to accurately quantify the obtained velocity field, which was not accomplishable by LSPT. Thus this study's results will help improve our knowledge on flame spreading over liquids. The following provides detailed comparison between two systems.

\section{COMPARISON BETWEEN LSPT AND PIV}

In our previously applied LSPT system, a high-speed camera with an ultraviolet lens and an image intensifier was used to obtain the velocity of seed particles. Ar-ion laser was used to measure gas-phase flow field and He-Ne laser to measure liquid-phase flow. Each laser formed roughly a $0.8 \mathrm{~mm}$ thick sheet of the continuous laser light. Superimposed image of liquid and gas phase was separated by a beam splitter and recorded by two digital video cameras. Seed particles recorded by digital cameras were tracked manually to obtain flow fields in both liquid and gas phases.

Our PIV system consists of two lasers that have pulse frequency of $15 \mathrm{~Hz}$, cross a correlation CCD camera with total 30 frames/s, a synchronizer, a video grabber, and computer software. The cross correlation camera has two images for each frame; the time delay between the two images $(25-32000 \mu \mathrm{s})$ can be controlled by the computer software and the synchronizer. PIV technique requires seeding for both air and liquid; hollow glass particles (8-12 $\mu \mathrm{m}$ diameter) for liquid and smoke particles $(1-2 \mu \mathrm{m})$ for air were used. Two laser pulses were sequenced to track trajectories of the seeding particles 
that were distributed in a 2-D laser illuminated plane. Figure 1 shows a schematic of our flame-spread apparatus and PIV system. Both top $(x-z)$ view and side $(x-y)$ view can be simultaneously obtained using two independent laser sheets. In this study, we focus 2-D flow data in order to compare it with our previous LSPT data, and show a schematic of 3D flow structure obtained by integrating those 2-D PIV flow data.

PIV system has an advantage over LSPT system having an integrated data recording and processing system that can replace a LSPT's painstaking manual tracking process. PIV system can track easily hundreds of particles, in comparison to the LSPT's less-than-50particle tracking capability making us possible to see more detailed flow structures than the LSPT. Because of the PIV's enhanced capability of tracking large number of particles combined with two pulse lasers and its automated data processing system, PIV system is expected to have better accuracy in measuring local flow fields than the LSPT system. By adjusting the sequence time of the PIV's two pulse lasers, a wide range of particle velocity can be accurately measured. Table 1 provides comparison between LSPT and PIV. The error shown in Table 1 was estimated based on the time resolution difference between the two systems.

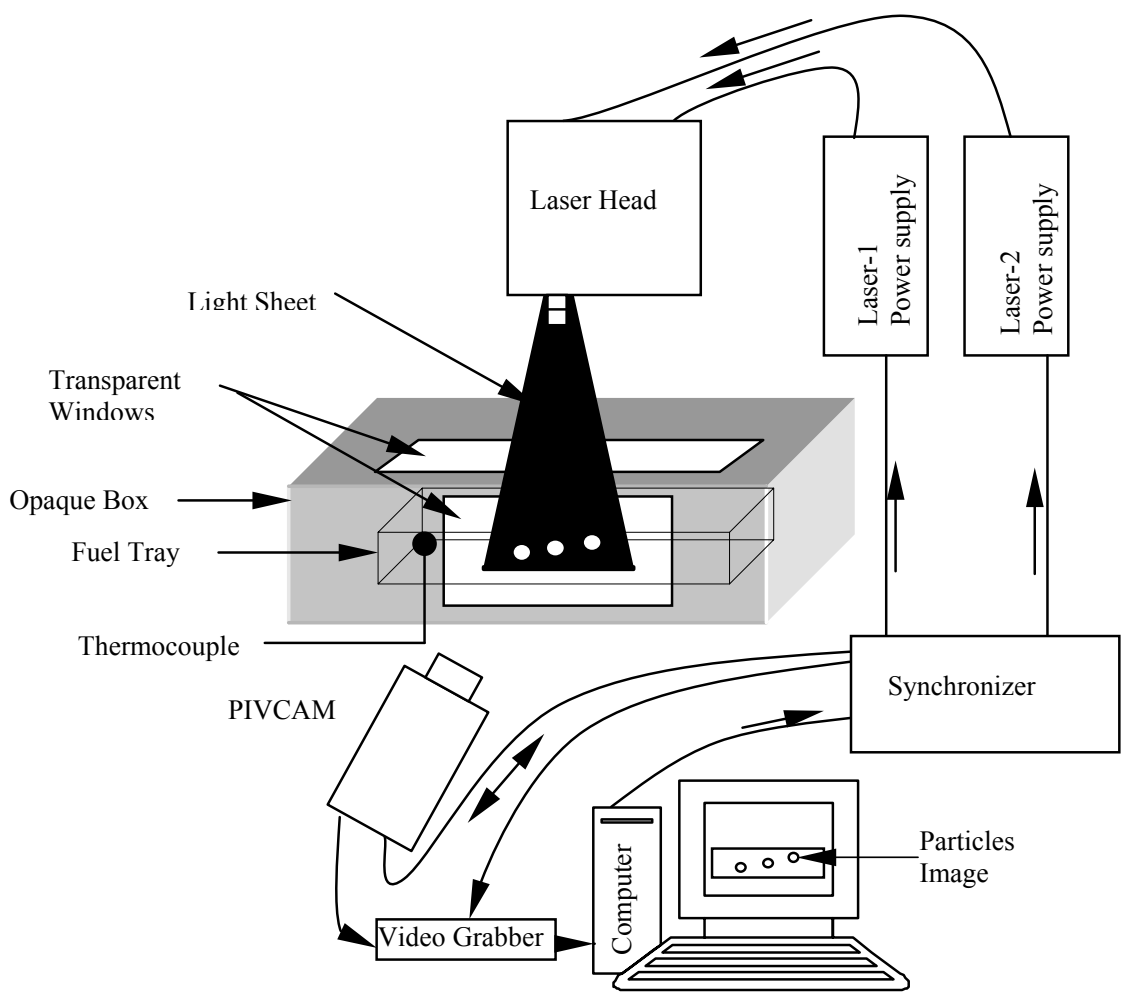

Fig. 1 - Flame spread apparatus and integrated particle image velocimetry. 
Table 1. Comparison of performance of LSPT to PIV

\begin{tabular}{l|l|l}
\hline \hline & LSPT [5] & PIV \\
\hline \hline Measurement capability & 2D with 3D applicability & 2D with 3D applicability \\
\hline Spatial resolution & $0.5 \mathrm{~mm}$ & $0.1 \mathrm{~mm}$ \\
\hline Time resolution & $33 \mathrm{~ms}$ & $25 \mu \mathrm{s}$ \\
\hline Particle tracking capability & $\begin{array}{l}\text { Manual process } \\
\text { (with up to 50 particles) }\end{array}$ & $\begin{array}{l}\text { Automated process } \\
\text { (easily hundreds particles) }\end{array}$ \\
\hline Estimated uncertainty & $10 \%$ & $1 \%$ \\
\hline
\end{tabular}

\section{PIV MEASUREMENTS}

To compare the current PIV results to our previous LSPT data, we employed the same liquid fuel tray that was used in our previous study (300mm long x $10 \mathrm{~mm}$ high $\times 20 \mathrm{~mm}$ wide). In our previous experiments, we employed several different liquid fuels including 1-propanol. Among them, 1-propanol fuel was chosen for our experiment because there is a good established database for comparison. The experimental procedure is clearly explained in our previous paper [5], so only summary is provided here.

1-propanol was placed in the tray just before the experiment and its initial temperature was measured by a $0.5 \mathrm{~mm}$-diameter type-K thermocouple that was placed just below the center of fuel surface. The initial air temperature in the opaque box is kept constant at 25 ${ }^{\circ} \mathrm{C}$. First, to see the general nature of spread behavior, the initial temperature of 1propanol was changed between $10{ }^{\circ} \mathrm{C}$ and $20^{\circ} \mathrm{C}$ in which temperature range the flamespread behavior changed from uniform spread to pulsating spread with a decrease of the liquid temperature. Then, we focused on PIV measurements at the $14{ }^{\circ} \mathrm{C}$ liquid temperature where pulsating spread occurs. The measured flame position, shown as a function of time in our previous paper [12], is not presented here. To achieve repeatable experimental condition, the fuel tray was enclosed with PMMA (transparent) case, 250 $\mathrm{mm}$ wide $\mathrm{x} 250 \mathrm{~mm}$ high $\mathrm{x} 400 \mathrm{~mm}$ long.

\section{RESULTS AND DISCUSSION}

Here we report our PIV data taken at two most critical steps in the pulsating flame spread process: just before and right after a flame jumping, the former corresponds to the Step [c] and the latter to Step [d] in our previous paper [5]. Figure 2 shows side view $(x-y$ plane) and top view ( $x-z$ plane) of flow fields in both gas and liquid phases measured by PIV and LSPT systems. The initial liquid temperature is $14{ }^{\circ} \mathrm{C}$, at which flame is in pulsating spread mode. At this step, just before a flame jumping, the flame is temporarily stopping and the upstream liquid fuel surface temperature is above the flash point (23 ${ }^{\circ} \mathrm{C}$ ), and the increase of fuel vapor concentration in the upstream is under way [5]. As shown in Figs. 2(ii) and (iii), PIV system detected both gas-phase and liquid-phase circulations, which are consistent with our LSPT results shown in Fig. 2(i).

As can be seen in these two figures, PIV revealed much more detailed flow structure than the LSPT results. An approximately $2 \mathrm{~mm}$-diameter recirculation cell was clearly shown in the gas phase, and in the liquid phase there was a large circulation whose center is located just ahead of the flame leading edge and approximately $2 \mathrm{~mm}$ deep. The similar gas phase cell was observed by Miller and Ross [10] during their flame spread experiments with concurrent airflow. Schiller et al. [9] predicted the existence of a small 
recirculation cell by numerical simulations. They explain that this recirculation cell is driven by a combination of buoyancy-driven flow opposed to the flame propagation direction and the flow in the same direction as flame spread induced by thermocapillarydriven motion at the liquid surface. Our PIV experiment confirmed that this gas-phase cell exists in the pulsating spread region. The concurrent airflow in Fig. 2(ii) is likely induced by the liquid surface flow, but not by the flame, because the velocity of the airflow decreased with an increase of the initial liquid temperature. In addition, the length of the concurrent airflow $(14 \mathrm{~mm})$ shown in front of the flame's leading edge is exactly the same as the length of the concurrent liquid surface flow. Figs 2(iv) and 2(v)

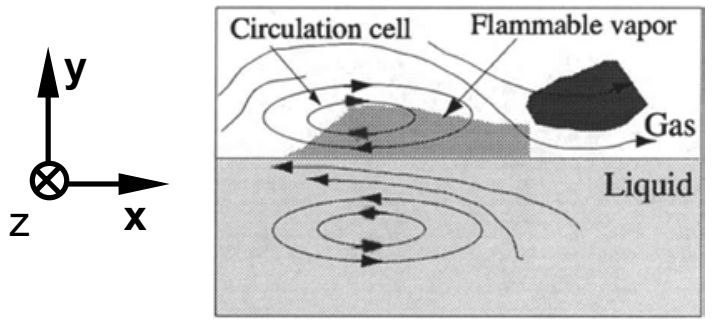

(i) Step (c) from Ref. [5] - side view

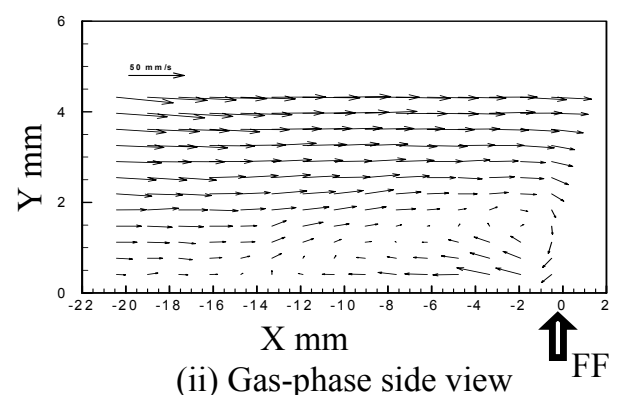

(ii) Gas-phase side view

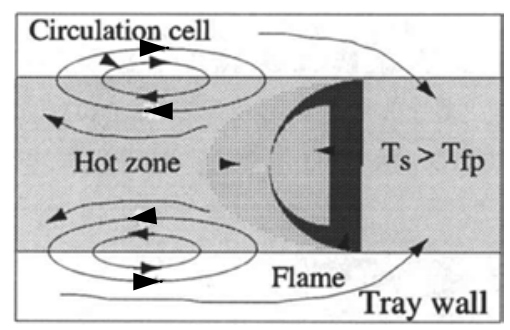

(iv) Step (c) from Ref. [5] - top view

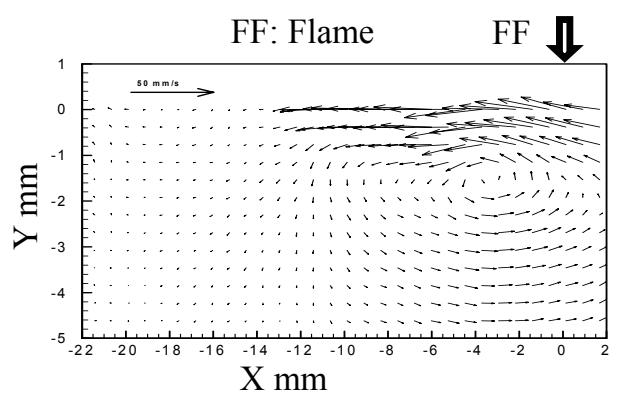

(iii) Liquid-phase side view

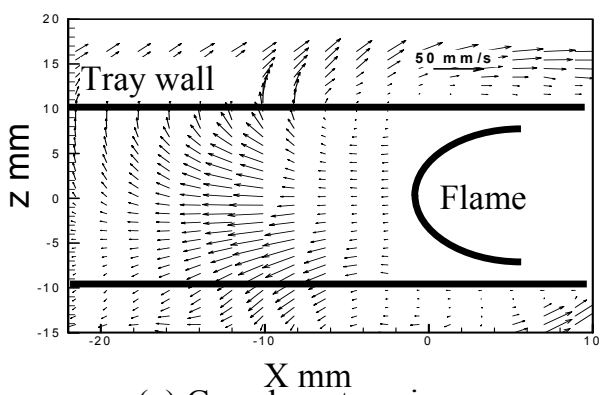

(v) Gas-phase top view

Fig. 2 - Comparison between LSPT and PIV velocity data just before a flame jumping. (i) step (c) from Ref. [5] (at $z=0$ ), (ii) PIV gas phase velocity profile in the $x-y$ coordinate (at $z=0$ ), (iii) PIV liquid phase velocity profile in the $x-y$ coordinate (at $z=0$ ), (iv) step (c) from Ref. [5] (at $y=1 \mathrm{~mm}$ ), (v) PIV gas phase velocity profile in the $x-z$ coordinate $($ at $y=1 \mathrm{~mm}$ ). 
show the top view ( $x-z$ plane) of the gas phase flow at $1 \mathrm{~mm}$ above the liquid surface obtained respectively by LSPT and PIV. PIV detected twin gas-phase circulation cells with more detailed flow structure than the LSPT results [5]. From the $x-y$ and the $x-z$ velocity profiles, we constructed a hypothesis of 3-D flow structure and show in Fig. 3.

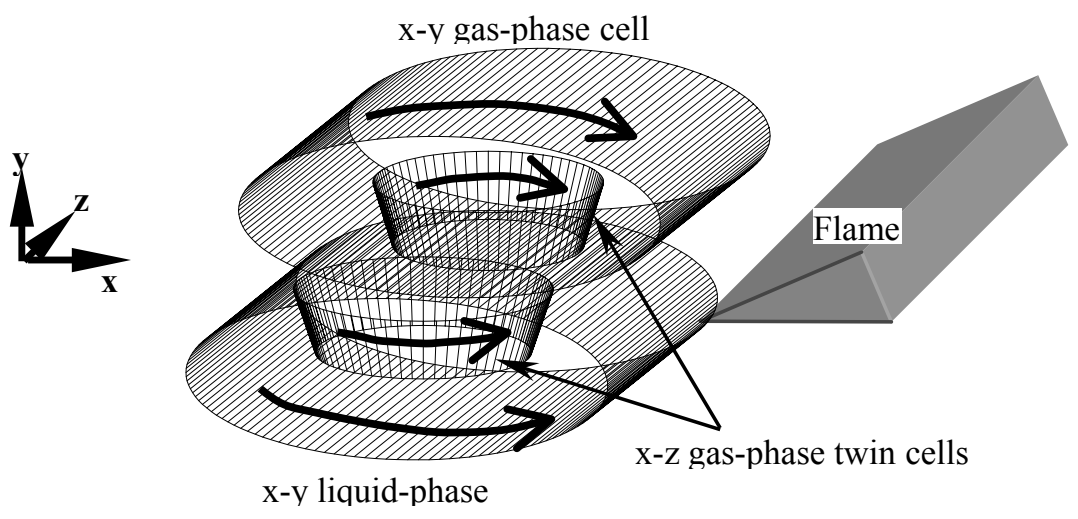

Fig. 3 - A schematic of 3-D (gas and liquid) velocity structure obtained by a series of 2-D PIV flow data.

Figure 4 shows flow structures at the flame-jumping step [5]. This critical step shows the flame jumping that occurs when the height of the flammable lean limit exceeds the quenching layer. At this step, the gas-phase does not have circulation. Because of a sudden increase of the flame spread rate associated with the flame jumping, the gas-phase circulation cell was destroyed due to thermal expansion, creating a new concurrent airflow. Our PIV result agrees with the LSPT result. However, in the liquid phase, PIV detected a small circulation that was not observed by the LSPT. We expected a circulation in the liquid phase when the flow is induced at the liquid surface to satisfy the mass balance. However, our previous LSPT measurement was insensitive to the liquid circulation just after the flame-jumping step due to its limited number of the seeded particles and time resolution. In addition, Konishi et al. [13] recently reported a small surface waveduring pulsating flame spread over ethanol suggesting the complixities of the pulsating flame spread mechanism.

\section{CONCLUSIONS}

We conducted a series of PIV velocity measurement experiments on flame spread over 1propanol which is under a pulsating flame spread condition. Our PIV data were then compared to our previous LSPT data. The following conclusions were obtained:

(1) At the step just before a flame jumping, both LSPT and PIV detected a twin vortex structure: one is at the gas phase circulating in the clockwise direction and the other is at the liquid phase circulating in the anti-clockwise direction. 
(2) At the step just after the flame jumping, PIV found a small circulation in the liquid phase and an irregular weak circulation in the gas phase, both of which were not reported in our previous LSPT experiments.

(3) Based on a series of 2-D PIV data, a complex 3-D structure of the flame induced flow was revealed and its schematic was shown.

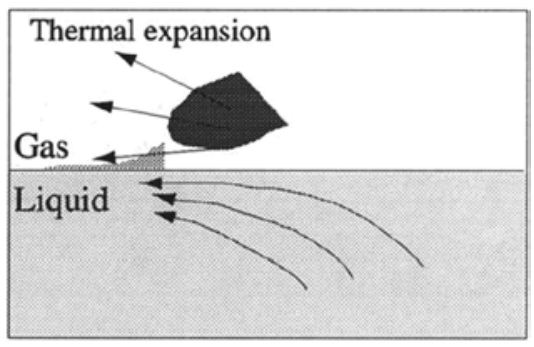

(i) Step (d) from Ref. [5] - side view

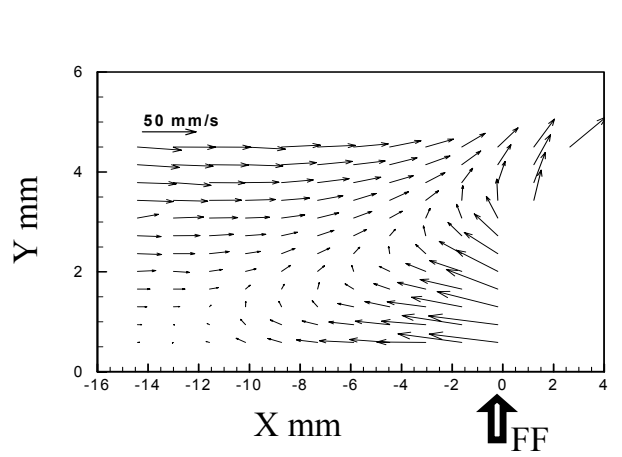

(ii) Gas-phase side view

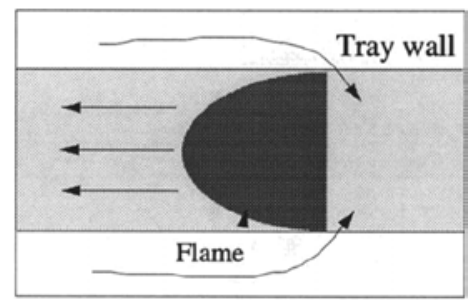

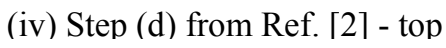

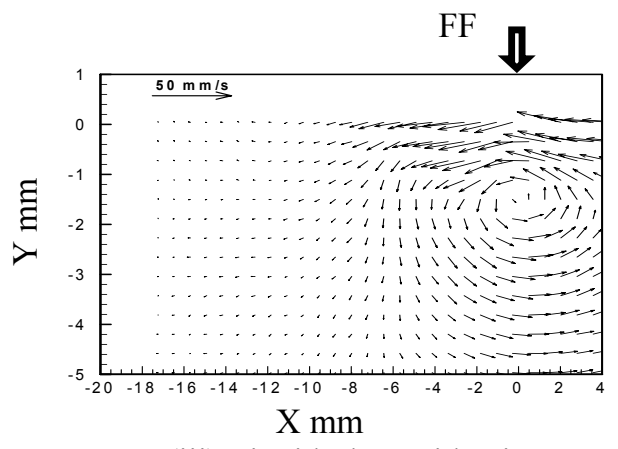

(iii) Liquid-phase side view

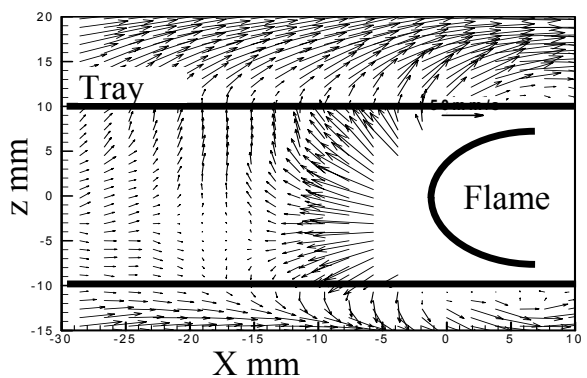

(v) Gas-phase top view

Fig. 4 - Comparison between LSPT and PIV velocity data just after a flame jumping. (i) step (d) from Ref. [5] (at $z=0$ ), (ii) PIV gas phase velocity profile in the $x-y$ coordinate (at $z=0$ ), (iii) PIV liquid phase velocity profile in the $x-y$ coordinate (at $z=0$ ), (iv) step (d) from Ref. [5] (at y=1 mm), (v) PIV gas phase velocity profile in the $\mathrm{x}-\mathrm{z}$ coordinate (at $\mathrm{y}=1 \mathrm{~mm}$ ). 


\section{ACKNOWLEDGEMENTS}

We would like to thank A. Ito and G. Tashtoush for many valuable discussions. This study was supported by Kentucky NASA-EPSCoR program and a special equipment grant from the University of Kentucky.

\section{REFERENCES}

1. Williams, F. A., Combustion Theory, Benjamin/Cummings, Menlo Park, CA, 1985, Chapter 12.

2. Ross, H. D., Prog. Energy Combust. Sci., 20, 17-63, 1994.

3. Ito, A., Masuda, D. and Saito, K., " A Study of Flame Spread Over Alchohols Using Holographic Interferometry,” Combustion and Flame, 83: 375-389, 1991.

4. Konishi, T., Naka, S., Ito, A., Saito, K., "Transient Two-Dimensional FuelConcentration Measurement Technique," Applied Optics, 36:.8815, 1997.

5. Konishi, T., Tashtoush, G., Ito, A., Narumi, A. and Saito, K., "The effect of Cold Temperature Valley on Pulsating Flame Spread Over Propanol," 28th Symposium (International) on Combustion, The Combustion Institute, 2819-2826, 2000.

6. Akita, K., "Some Problems of Flame Spread Along a Liquid Surface," 14th Symposium (International) on Combustion, The Combustion Institute, 1075-1083, 1972.

7. Torrance, K. E., "Subsurface Flows Preceding Flame Spread Over a Liquid Fuel," Combustion Science and Technology, 3: 133, 1971.

8. Sirignano, W. A., "A Critical Discussion of Theories of Flame Spread Across Solid and Liquid Fuels," Combustion Science and Technology, 6: 95, 1972.

9. Schiller, D. N., Ross and Sirignano, W. A., "Computational Analysis of Flame Spread Across Alcohol Pools," Combustion Science and Technology, 118: 205, 1996.

10. Miller, F. J. and Ross, H. D., "Smoke Visualization of the Gas-Phase Flow During Flame Spread Across a Liquid Pool," 27th Symposium (International) on Combustion, The Combustion Institute, 2715-2722, 1998.

11. Santro, R., Fernandez-Pello, A., Dryer, F., and Glassman, I., "Application of a TwoComponent LDV to the Measurement of Flows Induced by Flames Propagating Over Condensed Fuels," Applied Optics, 17: 3843, 1978.

12. Hassan, M. I. and Saito, K., "Propagation Characteristics of Flame Spread Over Liquid Fuels," Central States Section of The Combustion Institute, Spring Technical Meeting, April 16-18, 2000.

13. Konishi T., Ito, A., Kudou, Y., and Saito, K., "The Role of Flame-Induced Liquid Surface-Wave on Pulsating Flame Spread," accepted, $29^{\text {th }}$ Symposium (International) on Combustion, The Combustion Institute, in press, 2002. 